\title{
Functional Dissociation of Hippocampal Mechanism during Implicit Learning Based on the Domain of Associations
}

\author{
Michael Rose, ${ }^{1}$ Hilde Haider, ${ }^{2}$ Neda Salari, ${ }^{1}$ and Christian Büchel ${ }^{1}$ \\ ${ }^{1}$ NeuroImage Nord, Department for Systems Neuroscience, University Medical Center Hamburg Eppendorf, D-20246 Hamburg, Germany, and ${ }^{2}$ Institute of \\ Psychology, University of Cologne, 50931 Cologne, Germany
}

\begin{abstract}
Traditionally, the medial temporal lobe (MTL) was linked to explicit or declarative memory in associative learning. However, recent studies have reported MTL involvement even when volunteers are not consciously aware of the learned contingencies. Therefore, the mechanism of the MTL-related learning process cannot be described sufficiently by the explicit/implicit distinction, and the underlying process in the MTL for associative learning needs a more functional characterization. A possible feature that would allow a functional specification also for implicit learning is the nature of the material that is learned. Given that implicit memory tasks often comprise a combination of perceptual and motor learning, we hypothesized that implicit learning of the perceptual but not the motor component entails MTL activation in these studies. To directly test this hypothesis, we designed a purely perceptual and a purely motor variant of the serial reaction time task. In two groups of human volunteers, behavioral results clearly showed that both variants were learned without awareness. Neuronal recordings using fMRI revealed that bilateral hippocampal activation was observed only for implicit learning of the perceptual sequence, not for the motor sequence. This dissociation clearly shows that the functional role of the hippocampus for learning is determined by the domain of the learned association and that the function of the medial temporal lobe system is the processing of contingencies between perceptual features regardless of the explicit or implicit nature of the ensuing memory.
\end{abstract}

\section{Introduction}

In recent years, the exclusive role of medial temporal lobe (MTL) structures for explicit memory has been challenged by several studies that convincingly demonstrated involvement of the MTL also for implicit memory (Chun and Phelps, 1999; Rose et al., 2002; Henke et al., 2003a,b; Schendan et al., 2003; Degonda et al., 2005). This resulted in the need for a new definition of the MTLrelated learning processes independent of the explicit/implicit characterization to address the mechanism more directly (Cohen et al., 1997; Eichenbaum, 2001; Henke, 2010).

A classical task for the examination of implicit learning is the serial reaction time (SRT) task (Nissen and Bullemer, 1987), in which volunteers have to press keys that correspond to the location of the target stimuli. The sequence of stimuli is deterministic and can be learned without explicit knowledge about the sequence. Neuroimaging studies reported controversial results regarding the participation of MTL structures during SRT learning. Most studies observed activation in the putamen and the striatum (Grafton et al., 1995; Rauch et al., 1995; Berns et al., 1997; Hazeltine et al., 1997; Peigneux et al., 2000; Willingham et al.,

Received June 15, 2011; revised July 22, 2011; accepted July 29, 2011.

Author contributions: M.R., H.H., and C.B. designed research; M.R. and N.S. performed research; M.R. and N.S. analyzed data; M.R., H.H., and C.B. wrote the paper.

This work was supported by grants from the Deutsche Forschungsgemeinschaft.

Correspondence should be addressed to Dr. Michael Rose, Neurolmage Nord, Department for Systems Neuroscience, University Medical Center Hamburg Eppendorf, Martinistrasse 52, D-20246 Hamburg, Germany. E-mail: rose@uke.uni-hamburg.de.

DOI:10.1523/JNEUROSCI.3020-11.2011

Copyright $\odot 2011$ the authors $\quad 0270-6474 / 11 / 3113739-07 \$ 15.00 / 0$
2002). However, a recent study demonstrated MTL activation during implicit and explicit SRT learning (Schendan et al., 2003).

The controversial results may be explained by the fact that in the classical version of the SRT task, several different associations from different domains are confounded. Due to the fact that each target stimulus is associated with a distinct response, the motor and perceptual sequences of targets are perfectly correlated, and activation patterns observed in the classic SRT cannot be attributed to either domain. Furthermore, several combinations of associations across the domains were discussed to be important for learning the SRT, like learning the association of a response and the following stimulus (Ziessler and Nattkemper, 2001; Stöcker et al., 2003). Thus, only a decorrelation of the perceptual and motor contingencies would allow the separation of neural systems involved in each learning process and would allow a clear functional assignment. Previous results suggest a specific ability of the MTL for the processing of perceptual features, and therefore, it is conceivable that the function of the MTL in implicit learning is limited to the learning of perceptual associations.

To test this hypothesis, we designed a novel SRT task in which a trial-by-trial remapping of the stimuli to the response allows an independent manipulation of the perceptual or response sequence with an uncorrelated sequence in the opposite domain (see Fig. 1). Material including a perceptual color sequence was presented to one group of volunteers, and a second group practiced an identical task with an embedded motor response sequence (without any perceptual contingency). Due to the fact that the only difference between the two groups was the presence of different contingencies embedded in the same material, the comparison of learning-related effects between groups allowed 
us to test for a functional specialization of the MTL during implicit learning based on the nature of the material that includes the contingency.

\section{Materials and Methods}

Overall, 48 healthy volunteers (age 19-36, 25 female) participated in the fMRI experiment; 24 were assigned to the perceptual sequence learning group and 24 to the response sequence learning group (the sample was reduced to $12 / 15$ due to the exclusion of possible explicit participants; see Results). All subjects were right handed and had normal or corrected-tonormal vision. The study was approved by the ethics committee, and all participants gave written informed consent before participating in the study.

Stimuli. The colored squares $\left(0.4^{\circ} \times 0.4^{\circ}\right.$; maximal distance $2.3^{\circ}$ ) were presented on a gray background controlled by a PC that ensured synchronization with the MR scanner using the software "Presentation" (http://www. neurobs.com/). For the MR experiment, an LCD projector projected the stimuli on a screen positioned on top of the head coil, which was viewed by the subjects through a mirror $\left(10 \times 15^{\circ}\right.$ field of view). Participants entered the responses by pressing buttons on two MR-compatible devices (three buttons for each hand).

Design. In the employed experimental design, the participants in both groups practiced the identical task with an identical visual layout. In each trial, volunteers were shown six colored squares (red, green, magenta, black, yellow, and blue), and the location of each square was assigned to the corresponding finger button. The target square simultaneously appeared at fixation, and its color indicated the button that should be pressed in the actual trial (see Fig. 1). Each stimulus arrangement was presented for a maximum of $2 \mathrm{~s}$. It disappeared after the response was made, and after $300 \mathrm{~ms}$, the next trial started. Importantly, in each trial the assignment of the colors to the response locations varied. Therefore, only the location, not the color of the square, was assigned to a distinct response. This allows the generation of independent sequences for the color of the target stimulus (perceptual sequence learning) and response buttons (motor sequence learning). For the perceptual sequence group, the color of the target square changed across trials according to an underlying structured sequence (yellow, black, green, red, magenta, blue), but the order of responses was randomly assigned and not sequenced. In contrast, for the motor sequence group, the responses were structured (finger 5, 4, 2, 1, 6, 3), but the colors of the squares changed unpredictably. Stimulus presentation was guided by predefined input files that ensured that target color and responses were completely uncorrelated for both sequences (mean $r=0.05$, n.s., $\max r=0.08$ ).

In both groups, sequenced and random materials were presented in alternating blocks consisting of 22 trials (block duration $\sim 15 \mathrm{~s}$ ). In principle, the alternating presentation of random and sequenced blocks is similar to a probabilistic sequence of stimuli. The volunteer was not informed whether a random or a sequenced block was presented. Therefore, sometimes one color followed a distinct other color, and sometimes it did not. Between blocks, a fixation cross was presented for 8-12 s.
Motor Sequence Perceptual Sequence
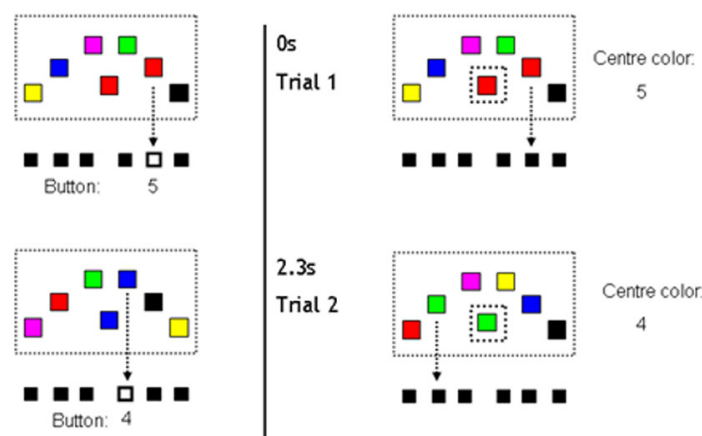

$2.3 \mathrm{~s}$

Trial 2
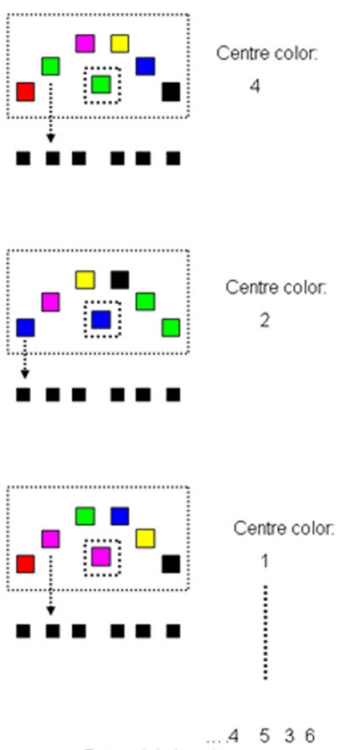

Deterministic color sequence

Button sequence: random
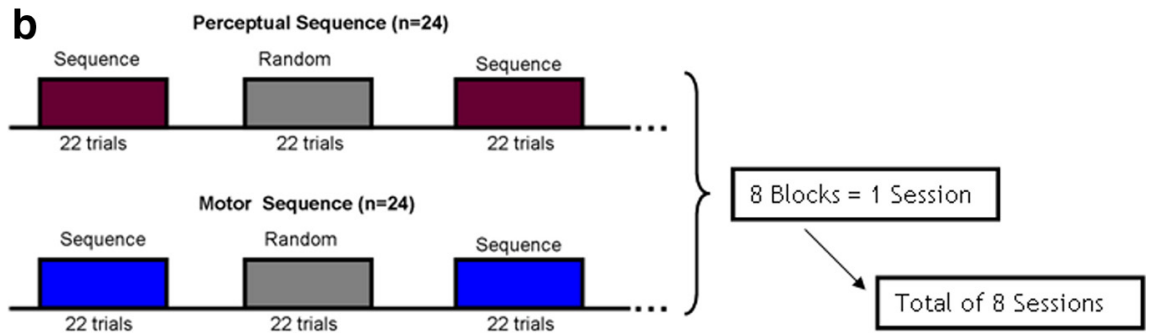

Figure 1. Task and design. In the task (a), six colored squares (red, green, magenta, black, yellow, and blue) were presented around the center, the locations of each square was assigned to a corresponding button. The target square in the middle simultaneously appeared at fixation and its color indicated the button that should be pressed in the actual trial (i.e., red in the center the finger that belongs to the red square i.e., the second finger of the right hand). Importantly, in each trial the assignment of the colors to the response locations changed, which allowed the independent establishment of a perceptual sequence (systematic variation of target color across trials) and a motor sequence (systematic variation of finger presses) without isual layout, but in one group a perceptual sequence and in the other group a motor sequence was embedded. In each session, 8 blocks with 22 trials each ( 4 random, 4 sequential in alternating order) were presented, and each volunteer practiced 8 sessions (704 random trials and 704 sequential trials).

Overall, eight learning sessions were presented, and each session comprised four random and four sequenced blocks.

The amount of possible explicit knowledge was estimated after the learning session using a completion task in combination with a wagering task (Persaud et al., 2007). The task was identical to the training session, except that only sequential material was used, the task was interrupted several times (each transition in the sequence four times), and the volunteers were asked to guess about the next response. Instead of a colored square, a question mark was presented in the center and then the volunteers had to guess about the next response by a button press in relation to the colored squares in the periphery. After each guess, the confidence of the response was assessed by a wagering task. The volunteer had to place a wager on the correctness of their response [ 50 cents for high confidence (a button press of the left hand) or 1 cent for low confidence (a button press of the right hand)]. 
Imaging. Functional MRI was performed on a $3 \mathrm{~T}$ system (Siemens Trio) with a gradient-echo EPI $\mathrm{T}_{2}{ }^{*}$ sensitive pulse sequence in 39 axial slices ( $2 \mathrm{~mm}$ thickness with $1 \mathrm{~mm}$ gap, TR $2.5 \mathrm{~s}$, TE $30 \mathrm{~ms}$, flip angle $80^{\circ}$, field of view $208 \mathrm{~mm}^{2}$, matrix $64 \times 64$ ). SPM8 (http://www.fil.ion.ucl. ac.uk/spm) was used for image processing and statistical analysis. All volumes were realigned to that first acquired, spatially normalized to the standard EPI template, and smoothed with a $6 \mathrm{~mm}$ FWHM isotropic Gaussian kernel.

The fMRI data were analyzed by an estimation of the BOLD signal for each condition (random/sequence) in each session (1-8), modeled as a hemodynamic response function with the duration of each block of stimuli. Using a general linear model, regression coefficients were obtained for each regressor (condition in session).

To test for the specificity of sequence learning in each group, the sequenced condition was compared with the random condition. This was statistically implemented at the level of the group analysis as an interaction contrast testing for an increase of activity across sessions limited to the sequenced condition (time $\times$ condition). Furthermore, the specificity of the learning effects in the perceptual learning group was tested against the learning effects in the motor sequence group as a three-way interaction of condition, session, and group. All contrasts were limited to voxels showing a reliable increase of activity across learning for the sequence condition (masking threshold $p<0.05$, uncorrected). All statistical tests were corrected for multiple comparisons [familywise error rate (FWE, $p<0.05$ ), region of interest analysis, $10 \mathrm{~mm}$ sphere centered around regions of interest for MTL and basal ganglia according to Schendan et al. (2003)].

\section{Results}

\section{Behavioral}

Assessment of awareness

After the learning sessions, the amount of possible explicit knowledge was estimated using a completion task. After each guess the confidence of the response was assessed by a wagering task. The volunteer had to place a wager on the correctness of their response (50 cents for high confidence or 1 cent for low confidence). The advantage of this wagering task is that even with implicit memory, above-chance performance could be achieved, but the lack of awareness would prevent in particular high wagers on correct responses (Persaud et al., 2007; Haider et al., 2010). Volunteers with scores above 20\% (chance level) correct highwagered response-indicating possible explicit knowledgewere excluded from further analyses. For the group with the perceptual sequence, a repeated-measures ANOVA [factors correctness and wager (high/low)] of the included participants showed a mean rate of correct high-wagered responses of only $7 \%, 86 \%$ incorrect answers, and no reliable difference between high and low wagers $\left(F_{(1,14)}=0.49\right.$, n.s. $)$. In the motor sequence group, $11 \%$ correct (high wagered) and $71 \%$ incorrect answers were observed with no difference between high and low wagers $\left(F_{(1,11)}=0.09\right.$, n.s. $)$. To rule out any potential source of explicit memory, additionally partial knowledge of separate parts of each sequence was examined. More than $50 \%$ correct responses for a single transition (i.e., red after blue, or finger 5 after 3 ) within the sequence also resulted in the exclusion from further analyses, even if the overall score was $<20 \%$.

Furthermore, the volunteers were asked whether they had noticed or felt any kind of regularity in the material, and no included participant noticed a regularity or was able to verbalize a typical sequence above chance level. Only one volunteer in the perceptual sequence group and three participants in the motor sequence group verbalized explicit knowledge. However, the rigorous criteria of the completion task and the examination of partial knowledge resulted in the exclusion of another eight volunteers from the perceptual and nine volunteers from the motor group.
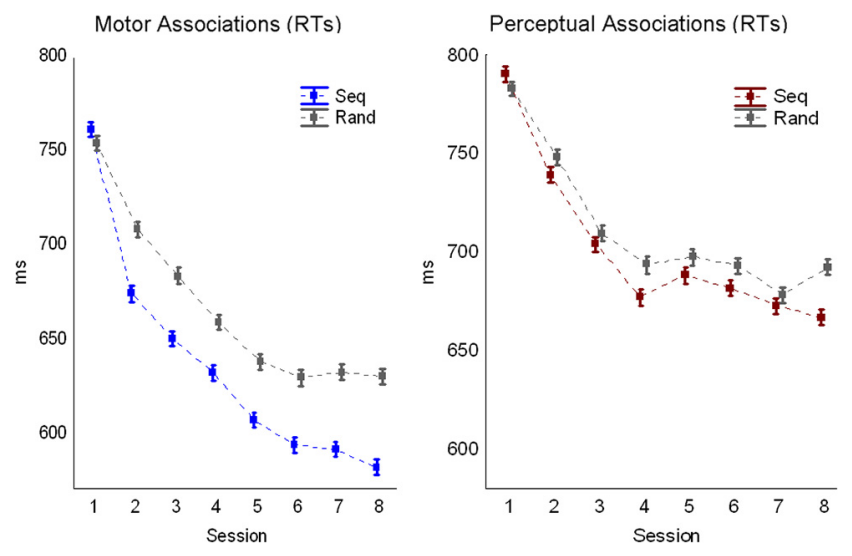

Figure 2. Behavioral results. The more pronounced decrease in response times across training for the sequential material in both groups demonstrates that over time sequential material is processed faster than the random material, indicating implicit learning of motor (blue) and perceptual (red) sequences [error bars indicating $95 \%$ confidence intervals for within-subject designs (Loftus and Masson, 1994)].

However, it should be noted that scores were small and did not necessarily reflect explicit memory of the whole sequence. However, by using the most conservative criteria, we assured the absence of any kind of explicit knowledge in the remaining sample ( $n=15$ for the perceptual sequence, $n=12$ for the motor sequence). All further results were estimated after the exclusion of these volunteers and therefore include only effects of implicit learning.

\section{Response times and errors}

In the remaining volunteers, error rate was low ( $\max 1.8 \%$ over all sessions) and did not differ between learning session or group. Mean response times (RTs) were calculated with respect to the onset of the correct response for each single input. For the motor sequence group, a repeated-measures ANOVA [factors session and condition (random/sequential)] revealed a general decrease of RTs across sessions $\left(F_{(7,77)}=60.5, p<0.001\right)$ and a difference between conditions $\left(F_{(1,11)}=42, p<0.001\right)$. Importantly, the interaction between session and condition $\left(F_{(7,77)}=12.7, p<\right.$ 0.001 ) showed that sequenced condition is processed faster than the random condition with practice indicating the specific acquisition of the sequential motor responses. For the perceptual sequence group, a general decrease of RTs $\left(F_{(7,98)}=24.5, p<\right.$ $0.001)$ and an interaction between session and condition $\left(F_{(7,98)}=\right.$ $11, p<0.001$ ) were observed. On closer inspection of the perceptual sequence group, a paired $t$ test further revealed reliably faster RTs for the sequential than for the random condition in the last block $\left(t_{(14)}=4.2, p<0.001\right)$ and also a main effect of condition when comparing the last five sessions $\left(F_{(1,14)}=16.7, p<0.001\right)$, showing that implicit knowledge about the color of the target stimulus could facilitate response selection.

A comparison of the learning effects between groups (repeated-measures ANOVA [within factors session and condition (random/sequential) and between factor group]) revealed a faster decrease of RTs for the motor sequence group (session $x$ group: $\left.F_{(7,175)}=4.2, p<0.001\right)$, most pronounced for the sequenced material in the motor group (condition $\times$ group: $\left.F_{(1,25)}=4.2, p<0.001\right)$ (Fig. 2). The three-way interaction of condition $\times$ session $\times$ group $\left(F_{(7,175)}=1.8\right.$, n.s. $)$ demonstrated only a trend toward a stronger expression of the learning in the motor sequence learning group. 
Table 1. Implicit learning effects for the perceptual sequence learning group

\begin{tabular}{|c|c|c|c|c|c|c|c|c|}
\hline \multirow[b]{2}{*}{ Region } & \multicolumn{4}{|c|}{ Implicit perceptual learning (time $\times$ condition) } & \multicolumn{4}{|c|}{ Specific for perceptual group (time $\times$ condition $\times$ group) } \\
\hline & $x$ & $y$ & $z$ & $t$ value & $x$ & $y$ & $\mathrm{Z}$ & $t$ value \\
\hline MTL hippocampus R & 28 & -24 & -18 & $3.98^{*}$ & 28 & -14 & -24 & $3.29 *$ \\
\hline MTL hippocampus L & -34 & -20 & -18 & $4.61^{*}$ & -32 & -22 & -22 & $3.49^{*}$ \\
\hline MTL hippocampus L & -30 & -10 & -22 & $3.47^{*}$ & -26 & -12 & -26 & $3.16^{*}$ \\
\hline MTL hippocampus/amygdala L & -26 & -6 & -23 & 3.06 & -24 & -6 & -24 & $3.74^{*}$ \\
\hline$B G$ ventral striatum $R$ & 10 & 12 & -10 & $4.43^{*}$ & & & & \\
\hline BG caudate $R$ & 18 & 12 & 10 & $4.28^{*}$ & & & & \\
\hline BG putamen $\mathrm{L}$ & -24 & 2 & -4 & $3.97^{*}$ & & & & \\
\hline
\end{tabular}

Within-group effects were assessed by the interaction contrast testing for increasing activity with learning for the sequence condition compared to the random condition. This interaction contrast was statistically compared with the identical contrast of motor sequence group. ${ }^{*} p<0.05$, corrected for multiple comparisons. BG, Basal ganglia.

Table 2. Implicit motor learning (time $\times$ condition) effects for the motor sequence learning group

\begin{tabular}{lrrrl}
\hline Region & \multicolumn{1}{l}{$x$} & $y$ & \multicolumn{1}{l}{$z$} & $t$ value \\
\hline BG R & 16 & 12 & -6 & $4.37^{*}$ \\
BG L & -22 & 6 & -4 & $3.63^{*}$ \\
Motor cortex R & 16 & -8 & 56 & 3.86 \\
Brainstem & 8 & -14 & -30 & 3.77 \\
Cerebellum R & 26 & -46 & -38 & 3.49 \\
\hline
\end{tabular}

Within-group effects were assessed by the interaction contrast testing for an increasing activity with learning for the sequence condition compared to the random condition. ${ }^{*} p<0.05$, corrected for multiple comparisons. BG, Basal ganglia.

\section{Neuroimaging results}

Learning-related activity was tested through a time by condition interaction, i.e., the contrast testing for a steeper increase of the fMRI signal across learning sessions for the sequence compared to the random condition. For the perceptual sequence group, activity increased across learning within the basal ganglia (including ventral striatum) and the MTL, including bilateral hippocampus (for details, see Table 1). For the motor sequence group, an increase of activity across sessions limited to the sequenced material (interaction time $\times$ condition) was found in the basal ganglia, motor cortex, and the cerebellum (for details, see Table 2) but not within the MTL, even at a very low threshold ( $p=0.05$, uncorrected).

To test for the specificity of these effects for perceptual learning, the interaction effect was compared with the identical contrast from the motor sequence group (Fig. 3). This three-way interaction (time $\times$ condition $\times$ group) showed a reliable effect restricted to bilateral hippocampus and demonstrated a learningrelated increase of activity only for implicit learning of a perceptual sequence and not for a motor sequence. Importantly, this interaction contrast controls for unspecific effects across time and also for the fact that the material in both groups is structured by an underlying sequence.

To further address possible group differences related to faster $\mathrm{RTs}$ in the motor sequence group, we compared the practice sessions of the random material between the two groups (different increase of neural activity across sessions). No reliable difference in the BOLD signal change with learning was observed for the comparisons that tested for a stronger increase of the BOLD signal observed for the random blocks of the motor sequence group or for the perceptual sequence group (whole brain, uncorrected $p<0.001)$. Thus, the processing of the random material did not result in differences between groups; i.e., the faster RTs in the motor sequence group did not affect the BOLD signal. Therefore, the results that were obtained for the interaction effects (mainly independent from general differences) can be regarded as specific for the underlying learning process.

To further evaluate the reliability of our results, another analysis method was used: for the last half of the training sessions, the

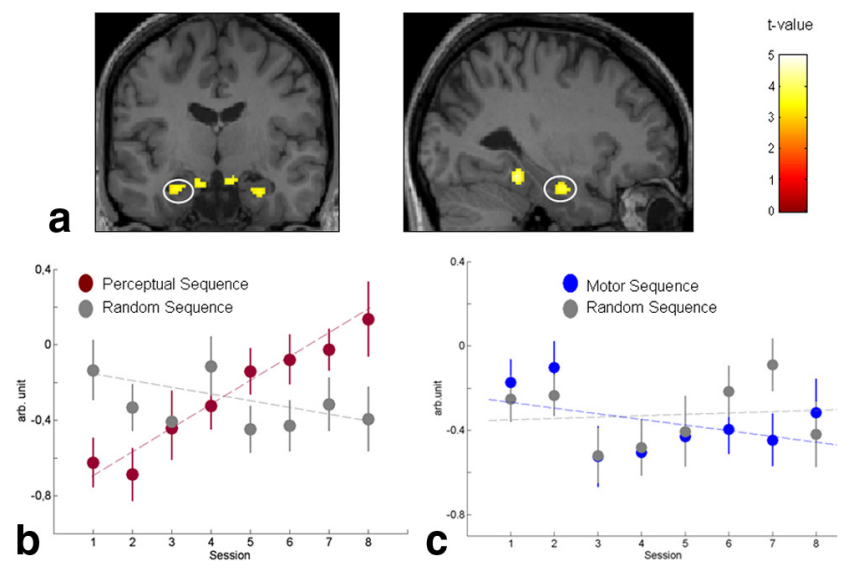

Figure 3. Implicit learning of a perceptual sequence. $\boldsymbol{a}$, fMRI effects were assessed by the three-way interaction of time $\times$ condition $\times$ group, showing increasing activity within bilateral hippocampus across learning only for the perceptual sequence and not for the motor sequence. For visualization, the percentage signal change of the peak voxel of the three-way interaction effect located in the left hippocampus $(-26,-12,-26)$ are plotted for each learning session and condition (random and sequenced material) for the group with the perceptual sequence $(\boldsymbol{b})$ and for the group with the motor sequence $(\boldsymbol{c})$.

main effect of the sequenced material against the random material was estimated for both groups (Fig. 4). For this period, the sequenced material resulted in an increased fMRI signal for the perceptual group in bilateral hippocampus (left: $x=-22, y=$ $-16, z=-22, t=3.1$; right: $x=22, y=-14, z=-14, t=3.1$ ) but not within the basal ganglia. In contrast, for the motor sequence group, a significant difference in response to the random material was observed in the basal ganglia (left: $x=-22, y=6$, $z=-4, t=3.7$; right: $x=16, y=12, z=-6, t=4.1)$ but not within the MTL. Again, this difference was evaluated statistically as an interaction of the two effects (sequenced material - random material $\times$ group), which showed a selective involvement of the hippocampus for perceptual sequence learning (left: $x=$ $-32, y=-22, z=-20, t=3.1)$ and an enhanced recruitment of the basal ganglia (right: $x=14, y=12, z=-4, t=3.2$ ) and motor cortex $(x=34, y=-24, z=52, t=4.1, p<0.001$ uncorrected) for motor sequence learning. Thus, in contrast to the time by condition effect, the result for the final half of learning demonstrated a stronger involvement of the basal ganglia and motor cortex for motor sequence learning. All statistical tests were corrected for multiple comparisons (FWE, $p<0.05$ ), with the identical region of interest analysis as the time $\times$ condition analyses.

\section{Discussion}

Limited to the perceptual domain implicit sequence learning resulted in learning-related activation within the MTL, more pre- 

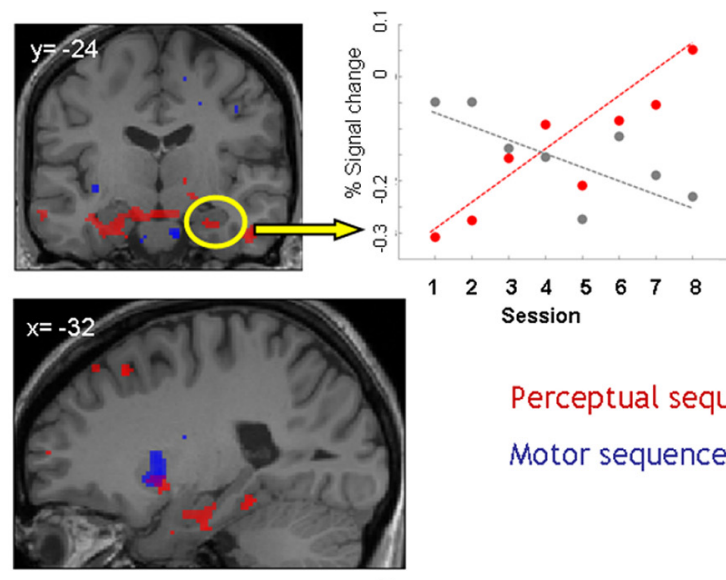

$\begin{array}{llllllll}1 & 2 & 3 & 4 & 5 & 6 & 7 & 8\end{array}$ Session

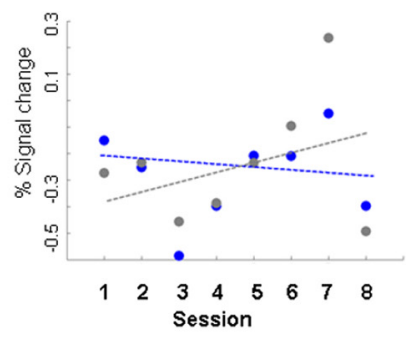

Perceptual sequence learning

Motor sequence learning
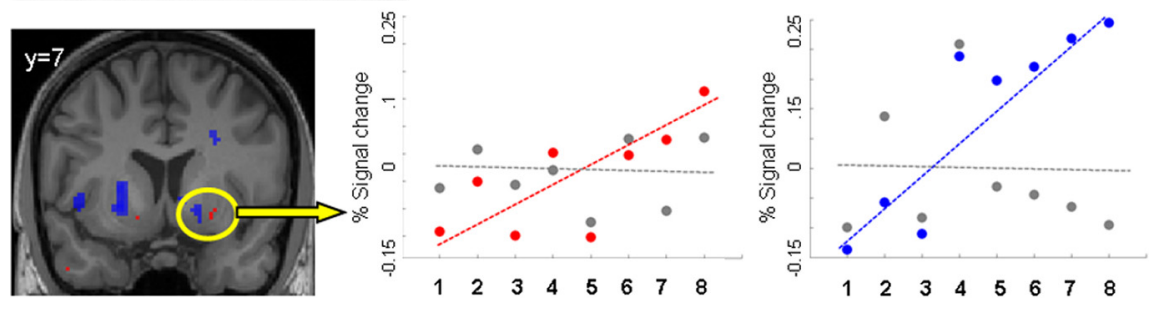

Figure 4. Effects for perceptual (red) and motor (blue) sequence learning. Both effects were estimated using the two-way interaction of time $\times$ condition for each group (display threshold: $p<0.001$, uncorrected, extent of 10 voxels). Activity within the hippocampus was selectively evoked by the perceptual learning process. Learning-related activity showed a small overlap within the ventral basal ganglia. The percentage signal change was extracted from key regions in the right hippocampus $(x=28 ; y=$ $-24 ; z=-18)$ and in the right basal ganglia $(x=-22 ; y=7 ; z=-4$; both using a sphere of $8 \mathrm{~mm})$.

This observation of implicit pure perceptual-based sequence learning is in accord with previous behavioral results (Remillard, 2003, 2009; Goschke and Bolte, 2007; Abrahamse et al., 2008, 2009). In most of the previous studies, volunteers implicitly learned a sequence of stimulus locations, which could entail the oculomotor system and therefore might also at least partially involve motor learning. However, in the present study, we can exclude possible eye-movement confounds, because the color sequence at fixation was not accompanied by regular locations of the color squares that indicated the response or a preparation of eye movements. Furthermore, the stimulus presentation ensured that in both groups the sequence of motor responses and target color stimuli was completely orthogonal. Thus, the only relation that could be learned in the perceptual sequence group was the association between the color stimuli at fixation, and this relation could not be expressed in terms of any motor responses.

Implicitly learning such a pure perceptualbased sequence resulted in an increasing fMRI signal across sessions in the hippocampus compared to the random blocks. This im-

cisely in bilateral hippocampus. In contrast, no effects were found in the MTL for response (i.e., motor) sequence learning. This finding is in agreement with the assumption that the function of the MTL is mainly determined by the detection of contingencies between perceptual features regardless of the explicit or implicit character of the memory. The present results demonstrate additional evidence that implicit learning can involve MTL structures if learning comprises perceptual contingencies and offer a functional differentiation of learning-related brain structures.

Specific learning-related effects were assessed by the comparison with the random material, i.e., without a sequence that can be learned. The intermixed presentation of random and sequenced blocks allows an estimation of the specific learning process at several stages and further resulted in probabilistic transitions between the stimuli, which prevents the generation of explicit memory. The rigorous criteria used to detect and exclude even partial explicit knowledge further assured that only volunteers with implicit memory were included in the analyses. The interaction effect between the sequenced and the random material with learning revealed specific learning-related activity and controls for unspecific effects like habituation or task practice and further allows a comparison between the two groups.

The behavioral results showed faster RTs for the sequential material in both groups with learning indicating the specific acquisition of the sequential knowledge. While this is a common and expected finding for motor response sequence learning, the reliable effect in the perceptual group is noteworthy. The implicit knowledge about the color of the next target stimulus provides no information about the selection of the correct response, because the experimental design ensured the absence of any correlations between the two domains. The faster RTs for the perceptual sequence thus indicate that the target stimulus processing can benefit from implicit knowledge on the color sequence. plicit learning effect within the MTL is in agreement with previous studies showing that the function of the MTL is not necessarily linked to participants' awareness (Chun and Phelps, 1999; Rose et al., 2002; Henke et al., 2003a,b; Schendan et al., 2003; Yang et al., 2003; Degonda et al., 2005; Hannula and Ranganath, 2009; Voss et al., 2009; Wang et al., 2010). However, the present experimental design extends this finding by a more precise functional characterization. The results of the motor sequence group show that it is not implicit learning in general that recruits MTL structures, but only those related to perceptual learning. In contrast, motor sequence learning involved the basal ganglia and motor cortex, which has previously been reported in SRT paradigms (Grafton et al., 1995; Rauch et al., 1995; Berns et al., 1997; Hazeltine et al., 1997; Peigneux et al., 2000; Willingham et al., 2002).

The specificity of the hippocampal responses for the perceptual sequence is clearly shown by the statistical comparison with the response sequence group. Although the visual input and the task were identical in the two groups, the three-way interaction demonstrated that the hippocampus was recruited during implicit learning only by material that incorporates a perceptual regularity and not for random material or material with a response sequence. Thus, the only difference that can explain the selective activation of the hippocampus is the embedded perceptual sequence.

Traditionally, the hippocampus has been linked to explicit memory processes and awareness (Squire and Zola-Morgan, 1991; Clark and Squire, 1998). However, it was previously shown that amnesic patients with lesions within the MTL were also impaired in implicit learning of contextual information, which was defined as a behavioral benefit from an adaptation to the spatial configuration and color of a visual search display (Chun and Phelps, 1999). The result was refined in a follow-up study using 
an identical task but different patient groups (Manns and Squire, 2001). In patients with a lesion that was largely confined to the hippocampal formation, no impairment of implicit learning was observed, but in patients with a lesion that extended to other structures of the MTL, this study also reported an influence on implicit context learning.

It was concluded that implicit learning was impaired only in patients with extensive damage to the MTL and that the hippocampus itself may be exclusively related to explicit memory. However, the present results and previous studies (Henke et al., 2003a,b; Schendan et al., 2003; Degonda et al., 2005) showed effects of implicit learning within the hippocampus proper. Further, the result of our study showed that the only reliable difference of the learning effects between the groups was located in bilateral hippocampus, indicating a functional relevance for the extraction of perceptual associations and not an epiphenomenon. This function is consistent with key properties of declarative memory as observed in humans, but does not limit this function to memory that can be assessed by awareness. A core function for the establishment of episodic or declarative memory is the integration of spatiotemporal information and the encoding of event sequences (Eichenbaum, 1997, 2000). Importantly, both processes can be conceptualized without the involvement of awareness. Recent theories suggested that memory is integrally tied to the various systems engaged during information processing; i.e., memory is a fundamental property of the operation characteristics of the human brain, and the memory systems should be described and dissociated on a functional basis (Brown and Aggleton, 2001; Eichenbaum, 2001; Bussey and Saksida, 2005; Montaldi and Mayes, 2010). MTL structures receive highly preprocessed sensory information and thus are ideally suited as coincidence detectors within the sensory domain (Suzuki and Amaral, 1994; Quiroga et al., 2005). Thus, the MTL provides a "convergence zone" to mediate associations (Marr, 1971). The present results demonstrated that even the sequence of colors can be learned and that this learning involved the hippocampus although the resulting memory was implicit. This result is in accord with the proposed role of the hippocampus for the formation of sequential knowledge (Devito and Eichenbaum, 2011) and with models that focused on the relational binding as the core memory process of the hippocampus independent of implicit or explicit forms of memory (Cohen et al., 1997).

Recently, a functional model for the hippocampus was proposed based on the information processing characteristics rather than on the conscious access (Henke, 2010). In this model, it was assumed that the hippocampus supports in particular the rapid encoding of flexible associations regardless of the explicit or implicit character. As an expansion of this model, the present results further show that the hippocampus also plays an important role for the extraction of perceptual contingencies during a slower implicit learning process that involves hundreds of learning trials. The limitation of this function to the perceptual domain supports a functional role of the hippocampus primarily based on the domain of the material that includes the learned contingencies.

In a previous study, we could show that the development of explicit memory during implicit learning can be regarded a separate process that controls the conscious access to the formed associations and that this process is not located within the MTL (Rose et al., 2010). In this study, explicit knowledge during an implicit learning process was associated with an increase in largescale connectivity and activity within prefrontal and ventral striatal areas, rather than within MTL. This emphasizes that hippocampal involvement is common across implicit and ex- plicit learning, whereas the explicit character of a memory relies on a process outside the MTL.

In summary, the present study showed that implicit learning of a color sequence involves the MTL and that this effect is purely driven by the perceptual contingencies. These results underline the dissociation of human memory systems based on the nature of learned information and argue against the view that memory systems are dissociated based on whether memories are explicit or implicit. Furthermore, this suggests a parsimonious system for learning of perceptual sequences that is common for implicit and explicit memory formation and only depends on the properties of the learned material.

\section{References}

Abrahamse EL, van der Lubbe RH, Verwey WB (2008) Asymmetrical learning between a tactile and visual serial RT task. Q J Exp Psychol (Colchester) 61:210-217.

Abrahamse EL, van der Lubbe RH, Verwey WB (2009) Sensory information in perceptual-motor sequence learning: visual and/or tactile stimuli. Exp Brain Res 197:175-183.

Berns GS, Cohen JD, Mintun MA (1997) Brain regions responsive to novelty in the absence of awareness. Science 276:1272-1275.

Brown MW, Aggleton JP (2001) Recognition memory: what are the roles of the perirhinal cortex and hippocampus? Nat Rev Neurosci 2:51-61.

Bussey TJ, Saksida LM (2005) Object memory and perception in the medial temporal lobe: an alternative approach. Curr Opin Neurobiol 15:730-737.

Chun MM, Phelps EA (1999) Memory deficits for implicit contextual information in amnesic subjects with hippocampal damage. Nat Neurosci 2:844-847.

Clark RE, Squire LR (1998) Classical conditioning and brain systems: the role of awareness. Science 280:77-81.

Cohen NJ, Poldrack RA, Eichenbaum H (1997) Memory for items and memory for relations in the procedural/declarative memory framework. Memory 5:131-178.

Degonda N, Mondadori CR, Bosshardt S, Schmidt CF, Boesiger P, Nitsch RM, Hock C, Henke K (2005) Implicit associative learning engages the hippocampus and interacts with explicit associative learning. Neuron 46:505-520

Devito LM, Eichenbaum H (2011) Memory for the order of events in specific sequences: contributions of the hippocampus and medial prefrontal cortex. J Neurosci 31:3169-3175.

Eichenbaum H (1997) Declarative memory: insights from cognitive neurobiology. Annu Rev Psychol 48:547-572.

Eichenbaum H (2000) A cortical-hippocampal system for declarative memory. Nat Rev Neurosci 1:41-50.

Eichenbaum H (2001) The hippocampus and declarative memory: cognitive mechanisms and neural codes. Behav Brain Res 127:199-207.

Goschke T, Bolte A (2007) Implicit learning of semantic category sequences: response-independent acquisition of abstract sequential regularities. J Exp Psychol Learn Mem Cogn 33:394-406.

Grafton ST, Hazeltine E, Ivry R (1995) Functional mapping of sequence learning in normal humans. J Cogn Neurosci 7:497-510.

Haider H, Eichler A, Lange T (2010) An old problem: how can we distinguish between conscious and unconscious knowledge acquired in an implicit learning task? Conscious Cogn 3:658-672.

Hannula DE, Ranganath C (2009) The eyes have it: hippocampal activity predicts expression of memory in eye movements. Neuron 63:592-599.

Hazeltine E, Grafton ST, Ivry R (1997) Attention and stimulus characteristics determine the locus of motor-sequence encoding. A PET study. Brain 120:123-140.

Henke K (2010) A model for memory systems based on processing modes rather than consciousness. Nat Rev Neurosci 11:523-532.

Henke K, Mondadori CR, Treyer V, Nitsch RM, Buck A, Hock C (2003a) Nonconscious formation and reactivation of semantic associations by way of the medial temporal lobe. Neuropsychologia 41:863-876.

Henke K, Treyer V, Nagy ET, Kneifel S, Dürsteler M, Nitsch RM, Buck A (2003b) Active hippocampus during nonconscious memories. Conscious Cogn 12:31-48.

Loftus GR, Masson ME (1994) Using confidence intervals in within-subject designs. Psychon Bull Rev 1:476-490. 
Manns JR, Squire LR (2001) Perceptual learning, awareness, and the hippocampus. Hippocampus 11:776-782.

Marr D (1971) Simple memory: a theory for archicortex. Philos Trans R Soc Lond B Biol Sci 262:23-81.

Montaldi D, Mayes AR (2010) The role of recollection and familiarity in the functional differentiation of the medial temporal lobes. Hippocampus 20:1291-1314.

Nissen MJ, Bullemer P (1987) Attentional requirements of learning: evidence from performance measures. Cogn Psychol 19:1-32.

Peigneux P, Maquet P, Meulemans T, Destrebecqz A, Laureys S, Degueldre C, Delfiore G, Aerts J, Luxen A, Franck G, Van der Linden M, Cleeremans A (2000) Striatum forever, despite sequence learning variability: a random effect analysis of PET data. Hum Brain Mapp 10:179-194.

Persaud N, McLeod P, Cowey A (2007) Post-decision wagering objectively measures awareness. Nat Neurosci 10:257-261.

Quiroga RQ, Reddy L, Kreiman G, Koch C, Fried I (2005) Invariant visual representation by single neurons in the human brain. Nature 435:1102-1107.

Rauch SL, Savage CR, Brown HD, Curran T, Alpert NM, Kendrick A, Fischmann AJ, Kosslyn SM (1995) A PET investigation of implicit and explicit sequence learning. Hum Brain Mapp 3:271-286.

Remillard G (2003) Pure perceptual-based sequence learning. J Exp Psychol Learn Mem Cogn 29:581-597.

Remillard G (2009) Pure perceptual-based sequence learning: a role for visuospatial attention. J Exp Psychol Learn Mem Cogn 35:528-541.

Rose M, Haider H, Weiller C, Büchel C (2002) The role of medial temporal lobe structures in implicit learning. An event-related FMRI study. Neuron 36:1221-1231.
Rose M, Haider H, Büchel C (2010) The emergence of explicit memory during learning. Cereb Cortex 20:2787-2797.

Schendan HE, Searl MM, Melrose RJ, Stern CE (2003) An FMRI study of the role of the medial temporal lobe in implicit and explicit sequence learning. Neuron 37:1013-1025.

Squire LR, Zola-Morgan S (1991) The medial temporal lobe memory system. Science 253:1380-1386.

Stöcker C, Sebald A, Hoffmann J (2003) The influence of response-effect compatibility in a serial reaction time task. Q J Exp Psychol A 56:685-703.

Suzuki WA, Amaral DG (1994) Perirhinal and parahippocampal cortices of the macaque monkey: cortical afferents. J Comp Neurol 350:497-533.

Voss JL, Hauner KK, Paller KA (2009) Establishing a relationship between activity reduction in human perirhinal cortex and priming. Hippocampus 19:773-778.

Wang WC, Lazzara MM, Ranganath C, Knight RT, Yonelinas AP (2010) The medial temporal lobe supports conceptual implicit memory. Neuron 68:835-842.

Willingham DB, Salidis J, Gabrieli JD (2002) Direct comparison of neural systems mediating conscious and unconscious skill learning. J Neurophysiol 88:1451-1460.

Yang J, Weng X, Guan L, Kuang P, Zhang M, Sun W, Yu S, Patterson K (2003) Involvement of the medial temporal lobe in priming for new associations. Neuropsychologia 41:818-829.

Ziessler M, Nattkemper D (2001) Learning of event sequences is based on response-effect learning: further evidence from a serial reaction task. J Exp Psychol Learn Mem Cogn 27:595-613. 